\title{
Bone Grafts in Periodontics-A Review
}

\author{
Pramod Tatuskar', Shobha Prakash²
}

Assistant Professor ${ }^{1}$, Vice principal, Prof \& HOD ${ }^{2}$

Department of Periodontics, College of Dental Sciences, Davangere

\section{Abstract:}

The ultimate goal of periodontal therapy should not be limited to the establishment and maintenance of periodontal health. The potential for regeneration of the hard and soft periodontal tissues lost to disease should be considered. Of all the bone grafting materials being developed, the demineralized freeze dried bone allograft (DFDBA) has been used as a substitute for bone graft for more than four decades. The basis for the use of any bone grafting material is to induce bone formation. In this article various bone grafts and biomaterials used are reviewed.

Keywords: Allograft, Autograft, Biomaterial, Bone graft.

\section{Introduction}

Bone grafts are used as a filler and scaffold to facilitate bone formation and promote wound healing. These grafts are bioresorbable and have no antigen-antibody reaction. These bone grafts act as a mineral reservoir which induces new bone formation. Bone grafting is a surgical procedure that replaces missing bone with material from patient's own body, an artificial, synthetic, or natural substitute. Bone grafting is possible because bone tissue has the ability to regenerate completely if provided the space into which it has to grow. As natural bone grows, it generally replaces the graft material completely, resulting in a fully integrated region of new bone. The upcoming of various bone grafts and biomaterials has given a big leap to the field of Periodontics. The aim of periodontal therapy is to control periodontal infection and inflammation in the periodontium. The continued function of the periodontally involved tooth requires additional periodontal support and regeneration of the lost periodontium ${ }^{1,2}$. This may be achieved by the use of guided tissue regeneration and various bone grafts and biomaterials used as substitutes of bone grafts. These are derived from vital or non vital bone sources or consist of organic orinorganic materials. The main aim of utilizing bone grafts and performing bone graft related osseous surgeries is to promote bone regeneration. However these materials should be biologically acceptable to the patient without causing any systemic or local hazards or side effects ${ }^{1}$.

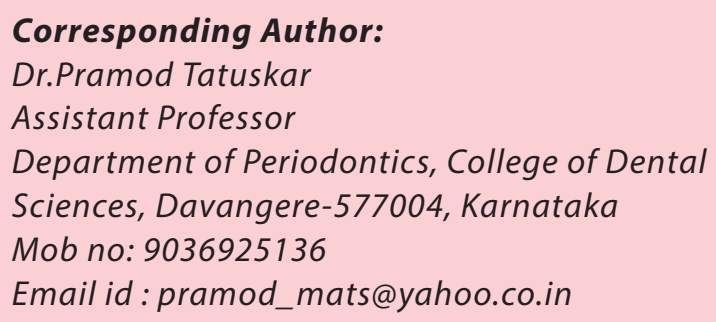

\section{BASIC CONSIDERATIONS: Definitions:}

1. Graft: A graft is a viable tissue that after removal from the donor site is implanted within the lost tissue which is then restored, repaired or regenerated ${ }^{3}$. Examples: Autografts, Allografts and Xenografts.

2. Biomaterials: These are bone derived materials processed in such a way that the cells are no longer vital and hence used as bone graft substitutes ${ }^{1}$.

Examples:- Freeze dried bone, decalcified or non decalcified.

- Xenograft from a bovine derived material can be more appropriately called

Xenogenic bone derived biomateria!

The biologic mechanisms that provide a rationale for bone grafting are osteoconduction, osteoinduction, and osteogenesis ${ }^{4}$.

\section{Osteoconduction :}

Occurs when bone graft material serves as a scaffold for new bone growth, which is perpetuated by the native bone. Osteoblasts from the margin of defect that is being grafted, utilize the bone graft material as a framework upon which to spread and generate new bone 5. In the very least, a bone graft material should be osteoconductive. 


\section{Osteoinduction :}

Involves stimulation of osteoprogenitor cells to differentiate into osteoblasts and then begins formation of new bone. The most widely studied type of osteoinductive cell mediators are BMPs. A bone graft material that is osteoconductive and osteoinductive will not only serve as a scaffold for

currently existing osteoblasts but will also trigger formation of new osteoblasts, promoting faster integration of the graft ${ }^{4}$.

\section{Osteopromotion :}

Involves enhancement of osteoinduction without possession of osteoinductive properties. For example, enamel matrix derivative enhances the osteoinductive effect of demineralized freeze-dried bone allograft (DFDBA), but will not stimulate bone growth alone ${ }^{4}$.

\section{Osteogenesis :}

It occurs when vital osteoblasts originating from bone graft material contributes to the growth of new bone along with bone formation.

The Ideal Requirements for bone grafts or bone graft substitutes may be summarised as follows ${ }^{1}$ :

1. Biologic acceptability

\section{Predictability}

\section{Clinical feasibility}

\section{Minimal operative hazard}

5. Minimal post operative sequelae

6. Patient acceptance.

Types and Tissue Sources:

Autograft :

Autologous or autogenous bone grafting involves utilizing bone obtained from same individual receiving the graft. Bone can be harvested from nonessential bones, such as from iliac crest, mandibular symphysis (chin area), and anterior mandibular ramus (coronoid process). When a block graft will be performed,autogeneous bone is the most preferred because there is less risk of graft rejection as the graft is originated from the patient's body ${ }^{6}$. It would be osteoinductive and osteogenic, as well as osteoconductive. Disadvantage of autologous grafts is that additional surgical site is required, another potential

location for postoperative pain and complications ${ }^{6}$. All bones require blood supply in the transplanted site. Depending on where the transplant site is and size of the graft, an additional blood supply may be required. For these types of grafts, extraction of the part of theperiosteum and accompanying blood vessels along with the donor bone is required. This kind of graft is known as a free flap graft.

\section{Allografts :}

Allograft is derived from humans. The difference is that allograft is harvested from an individual other than the one receiving the graft. Allograft bone is taken from cadavers that have donated their bone so that it can be used for living people who are in need of it; it is typically sourced from a bone bank. There are three types of bone allograft available ${ }^{1,7}$ :

\section{Fresh or fresh-frozen bone \\ 2. FDBA \\ 3. DFDBA}

The use of allografts for bone repair often requires sterilization and deactivation of proteins normally found in healthy bone. Contained in the extracellular matrix of bone tissue are the full cocktail of bone growth factors, proteins, and other bioactive materials necessary for osteoinduction and successful bone healing; the desired factors and proteins are removed from the mineralized tissue by using a demineralizing agent such as hydrochloric acid. The mineral content of the bone is degraded, and the osteoinductive agents remain in a demineralized bone matrix (DBM).

\section{Xenograft :}

Xenogratfs are bone grafts from a species other than human, such as bovine and are used as a calcified matrix. Alloplastic grafts :

Alloplastic grafts may be made from hydroxyapatite, a naturally occurring mineral (main mineral component of bone), made from bioactive

glass. Hydroxyapatite is a synthetic bone graft, which is the most used now due to its osteoconduction, hardness, and acceptability by bone. Some synthetic bone grafts are made of calcium carbonate, which start to decrease in usage because it is completely resorbable in short time and makes breaking of the bone easier. Finally used is the tricalcium phosphate in combination with hydroxyapatite and thus giving effect of both, osteoconduction and resorbability. 


\section{Synthetic variants :}

Flexible hydrogel-hydroxyapatite (HA) composite which has a mineral to organic matrix ratio, approximating that of human bone. Artificial bone can be created from ceramics such as calcium phosphates (e.g., HA and tricalcium phosphate), bioglass, and calcium sulphate are biologically active depending on solubility in physiological environment ${ }^{8}$. These materials combine with growth factors, ions such as strontium or mixed with bone marrow aspirate to increase biological activity. The presence of elements such as strontium can result in

higher bone mineral density (BMD) and enhanced osteoblast proliferation.

\section{Growth factors :}

Growth factors enhanced grafts are produced using recombinant DNA technology. They consist of either human growth factors or morphogens (BMPs in conjunction with a carrier medium, such as collagen). The factors and proteins that exist in bone are responsible for regulating cellular activity. Growth factors bind to receptors on cell surfaces and stimulate intracellular environment to act. Generally, this activity translates to a protein kinase that induces a series of events resulting in transcription of messenger ribonucleic acid (mRNA) and ultimately into the formation of a protein to be used intracellularly or extracellularly. The combination and simultaneous activity of many factors results in controlled production and resorption of bone. These factors, residing in extracellular matrix of bone, include TGF-beta, insulin like growth factors I and II, PDGF, FGF, and BMPs ${ }^{9,10}$ ,Cell-based bone graft substitutes: Stem cells are cultured in the presence of various additives such as dexamethasone, ascorbic acid, and b-glycerophosphate to direct the undifferentiated cell towards osteoblast lineage. The addition of TGF-beta and BMP-2, BMP-4, and BMP-7 to the culture media can also influence the stem cells towards osteogenic lineage. Mesenchymal stem cells have also been seeded onto bioactive ceramics conditioned to induce differentiation to osteoblasts ${ }^{8,11}$.

\section{BONE GRAFTS AND BONE GRAFT SUBSTITUTES(Table-1) :}

I) Non-osseous material :

A) Organic;-

\section{i) Dentin:}

Although demineralised dentin has been advocated as a possible graft ${ }^{12}$, it has not gained widespread acceptance because clinical results have been some what disappointing ${ }^{13}$.

\section{ii) Dura:}

The use of lyophilised allogeneic duramater in periodontal osseous defects has resulted in gain of clinical attachment and reduction in probing depth. Histologically bone has been seen alongside but never in the implant ${ }^{14}$.

\section{iii) Sclera:}

Success has been reported with preserved sclera ${ }^{15}$, but there were no signs of osteogenesis within the sclera and it remained encapsulated at the site ${ }^{13}$.

\section{iv) Cartilage:}

Cartilage allografts provide no statistically significant advantage in bone or soft tissue repair over flap debridement alone ${ }^{16}$.

TABLE 1: CATEGORIES OF BONE GRAFTS AND BONE GRAFT SUBSTITUTES

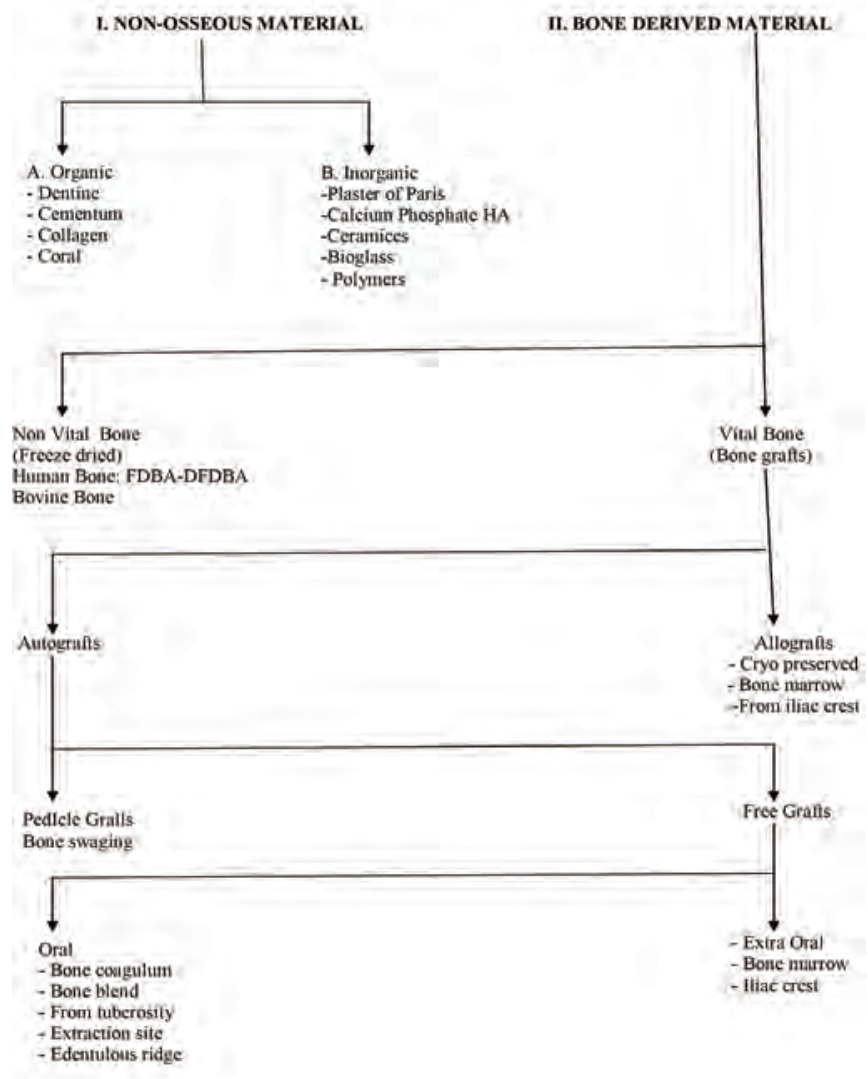




\section{B) Inorganic;-}

\section{i) Plaster of Paris:}

Plaster of Paris (Calcium sulphate) is biocompatible and porous thereby allowing fluid exchange, which prevents nap necrosis. Plaster of Paris resorbs completely in 1-2 weeks ${ }^{17}$. Also, because plaster of paris is so rapidly resorbed, it is questionable whether it is of any benefit.

\section{ii) Bio active glasses:}

There are 2 forms of bioactive glass currently available, perio glass (Bio glass synthetic bone graft particulate) and biogran (resorbable synthetic bone graft). Bio active glasses are composed of SI02, $\mathrm{CaO}, \mathrm{Na20}, \mathrm{P} 205$ and bond to bone is through the development of a surface layer of carbonate hydroxyapatite (HA). When exposed to tissue fluids invivo, the bioactive glass is covered by a double layer composed of silica gel and a calcium phosphate-rich (apatite) layer. This calcium phosphate-rich layer promotes adsorption and concentration of proteins used by osteoblasts to form a mineralised extracellular matrix. It is theorized that these bioactive properties guide and promote osteogenesis, allowing rapid and quick formation of new bone ${ }^{18}$.

\section{Bone derived material :}

\section{A) Intraoral autografts :}

\section{a) (i) Osseous coagulum and bone blend:}

Robinson, in 1969, having considered that the grafted particles of bone should be of smaller size and that mineralised fragments can induce osteogenesis, developed a technique, which he termed osseous coagulum, using the material evacuated during osseous surgery. The donor bone is obtained from any intra-oral source, usually areas requiring osseous correction. A number 6 or 8 round carbide bur, rotating at 25000 to $30000 \mathrm{rpm}$, without irrigation, produces bone dust which when coated with blood makes an osseous coagulum. Robinson showed the feasibility of the technique in several case reports with re- entries from 3 to 24 months. Diem et al in 1972, proposed the use of an amalgam trituration capsule and a pestle to reduce bone fragments to a workable, plastic like mass, called Bone Blend ${ }^{1}$. ii) Intra-oral cancellous bone marrow transplants:

Haitt and Schallhorn have described the use of cancellous bone obtained from the maxillarytuberosity, edentulous areas and healing sockets. The maxillary tuberosity frequently contains a good amount of cancellous bone, particularly if the third molars are absent. Foci of red marrow are also occasionally observed. Edentulous ridges can be approached with a flap, and cancellous bone and marrow are removed with curettes. Sockets are allowed to heal for 8-12 weeks, and the apical portion is utilised as donor material. The particles are reduced to small pieces ${ }^{15}$.

\section{b) Bone swaging:}

This technique requires the existence of an edentulous area adjacent to the defect, from which the bone is pushed into contact with the root surface without fracturing the bone at its base ${ }^{15}$.

\section{B) Iliac bone and marrow autografts:}

Schallhorn, in 1968, described the first autogenous iliac bone graft in one case report where a bone marrow biopsy was immediately implanted into interproximal bony craters between both lower canines and lateral incisors. At 5 months re-entry, the defects appeared completely filled. Autografts of iliac bone and marrow possess a high osteogenic potential, even in supra crestal bone fill and class II furcations. They are able to induce cementogenesis, bone regeneration and Sharpey's fibres reattachment with functionally oriented periodontal ligament. Fresh iliac bone and marrow are responsible for a high rate of resorption and ankylosis. The need for an extra oral surgical site and for a sampling techmque performed by a trained specialist represent serious limitations to this procedure ${ }^{1}$.

\section{BIOMATERIALS}

\section{(Table-2): I. Bone derived biomaterials}

\section{A. Freeze dried human bone :}

These are bone graft substitutes which are available through bone banks and are obtained from autopsy of human donors within 24 hours after death and are further lyophilized after a series of physical and chemical processing. In this procedure the guide lines of the American Association of Tissue Banks is followed ${ }^{1}$. It includes: 


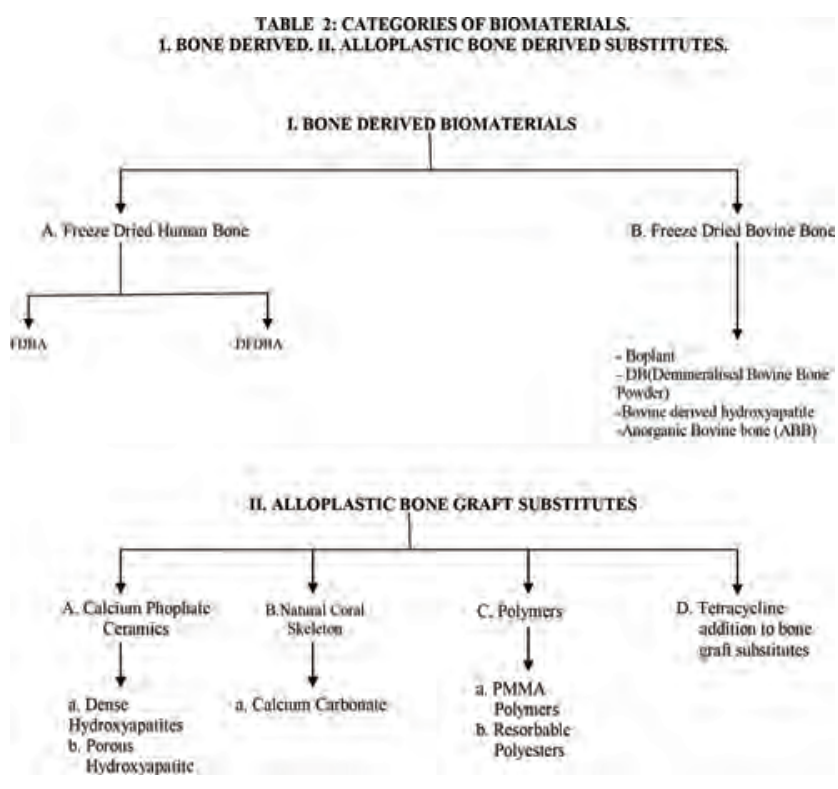

- Bone procured from the human donor within 24 hours after death.

- It is frozen to $-1790 \mathrm{C}$ for 4 weeks.

- Graft is freeze dried.

- Graft is defatted in chloroform menthol for 6 hours at $25^{\circ} \mathrm{C}$.

- Autodigested in phosphate buffers containing iodoacetate acid and sodium azide for 72 hours at $37^{\circ} \mathrm{C}$.

- Demineralized in 0.6 N HCL for 3 days at $4^{\circ} \mathrm{C}$.

- After each step bone is washed with triple distilled water.

- Refrozen and freeze dried.

- Ground under cryogenic conditions to yield a particle size of 125-1000 microns.

The osteo inductive capacity of these materials is governed by the processing procedure. The inductive activity gradually decreases atjd eventually is reduced to 0 within a period of 15 days when decalcification with $0.6 \mathrm{~N} \mathrm{HCL}$ is performed at $25^{\circ} \mathrm{C}$. wheareas in cold temperature $\left(2^{\circ} \mathrm{C}\right)$, the inductive activity is fairly maintained even at 30 days. Ethyl or isopropyl alcohols in $0.6 \mathrm{~N}$ HCL produces total inactivation of inductive substitute. Delipidization with ether, alcohol, acetone, hexachlorophene, common detergents and Tween 80 may even enhance bone induction. Heating above $60^{\circ} \mathrm{C}$ inhibits bone formation.

\section{B. Freeze Dried Bovine Bone :}

\section{i) Boplant :}

The use of Boplant, a bovine bone processed by detergent, chloroform and methanol extraction to reduce lipid content, washed, sterilized in antiseptic solution and lyophilized was first used way back in 19661 . The extensive usage of this material led to the conclusion that the material was incorporated by new bone formation, and it was accepted as a well tolerated, non resorbable, osteoconductive material. It was observed that the osteogenic potential of decalcified Boplant did not appear greater than that of mineralized Boplant ${ }^{1,19}$.

\section{ii) Demineralized bovine bone powder (DBP):}

It is prepared from cortical portions of bovine bone after 1 hour extraction in $100 \%$ ethanol and ethyl ether. It is then pulverized in liquid nitrogen mill, demineralized for 3 hours in $0.5 \mathrm{~N} \mathrm{HCI}$ and washed with water, absolute ethanol and anhydrous ether until neutral $\mathrm{pH}$ is achieved ${ }^{1}$

\section{iii) Bovine derived Hydroxyapatite :}

This is a xenogenic bone graft substitute, described as $100 \%$ crystalline, naturally porous, bovine derived hydroxyapatite. The use of this material was reported in two cases by Callan and Rohrer, in $1993{ }^{1}$.

\section{iv) Anorganic bovine bone (ABB):}

This is also a xenogenic variety, said to contain carbonate apatite. It is defatted and collagen is eluted. Though various studies were conducted by Thaller et al in 1993 and 1994 on animal models, there has been no study testing $A B B$ in human periodontal defects to date ${ }^{1}$.

\section{Risks of disease transmission :}

Transmission of blood borne and tissue borne disease has raised concern about allogenic bone graft substitutes ${ }^{1,13}$. The xenogenic bone has also recently been suspected of potentially transmitting prion diseases. AIDS and Creutzfeldt - Jakob diseases (CJD) are at risk of getting transmitted by allotransplantation and bovine spongiform encephalopathy (BSE) could get transmitted by bovine bone transplantation. Both BSE and CJD belong to a group of diseases affecting CNS in animals as well as in humans. 
Scrapie is the prototype of these diseases. It affects mainly sheep, while BSE, a new affects bovines. In human CJD, Gerstmann - Straussler - Sheinker (GSS) disease and Kurv represents the main form of this disease. These are characterized by excitability, itching, ataxia, paralysis and death. The diagnosis of this disease is by autopsy. At autopsy, the brain show spongiform changes, formation of amyloid plaques and accumulation of abnormal form of host encoded proteins.

\section{Prevention of HIV transmission:}

HIV can be transmitted to patients receiving kidney, heart and liver transplants, as well as skin grafts and bone grafts. Rigorous screening of potential donors by medical and social screening, HIV antibody test, HIV antigen test, autopsy and special lymph node examinations is critical. Buck et al 1989, have calculated that the risk could be as low as $0.00006 \% 1$. Mellonig et al 1992, have demonstrated that HIV infected bone graft is rendered free of virus contamination after the DFDBA processing ${ }^{1}$.

\section{Fate of Graft Material:}

Once the material is placed in the bony defect it may act in a number of ways which may decide the fate of the graft material ${ }^{2-20}$. The various possibilities include: Bone graft material may have no effect at all. The bone graft material may act as a scaffolding material for the host site to lay new bone. The bone graft material may itself deposit new bone because of its own viability.

\section{Conclusion :}

Therefore it is evident that Bone grafts and bio materials used' in Periodontal therapies have shown remarkable results as compared to the simple procedures like open curettage, and flap debridement surgery alone for periodontal osseous defects. At present, barrier techniques to prevent epithelial downgrowth, with or without autogenous bone or decalcified freeze-dried bone allograft, are widely used. Coral - derived materials can be used when non human derived grafts are preferred. Bone substitutes will definitely have an increased role to play in the future of periodontal regeneration.

\section{References :}

1. Jean-Pirr Olika Youn. Proceedings of the 2nd European workshop on Periodontics. In Niklous P. Lang, ed. Bone grafts and biomaterials used as bone graft substitutes. Berlin, Quintic ssenz Verlag, 1997-313.

2. Polson A.M. Periodontal regneration Current status and directins. 151 edition; Quintessence publishing Co., Inc 1995, 9-10: 71-112.

3. Grant, Stern, List garten, Periodontics. (6th edition). The C.V. Mosby Company 1988; 838-82.

4. Giannoudis PV, Dinopoulos H, Tsiridis E. Bone substitutes: An update Injury 2005;36(Suppl 3):S20-7.

5. Laurencin C, Khan Y, El-Amin SF. Bone graft substitutes. Expert Rev Med Devices 2006;3:49-57.

6. Conrad EU, Gretch DR, Obermeyer KR, Moogk MS, Sayers M, Wilson JJ,et al. Transimission of the hepatitis-C virus by tissue transplantation. J Bone Joint Surg Am 1995;77:214-24.

7. Centres for disease control and prevention. Septic arthritis following anterior cruciate ligament reconstruction using tendon allografts: Floridaand Louisiana, 2000. MMWR Morb Mortal Wkly Rep 2001;50:1081-3.

8. Centres for disease control and prevention (CDC ). Update: Allograft-associated bacterial infections: United States, 2002. MMWR Morb Mortal Wkly Rep 2002;51:207-10.

9. Valdes MA, Thakur NA, Namdari S, Ciombor $\mathrm{DM}$, Palumbo M. Recombinant bone morphogenic protein-2 in orthopaedic surgery: A review. Arch Orthop Trauma Surg 2009;129:1651-7.

10.Mulconrey DS, Birdwell KH, Flynn J, Cronen GA, Rose PS. Bone morphogenic protein (RhBMP-2) as a substitute for iliac crest bone graft in multilevel adult spinal deformity surgery: Minimum two-yearevaluation of fusion. Spine (Phila Pa 1976) 2008;33:2153-9.

11.Waked W, Grauer J. Silicates and bone fusion. Orthopedics 2008;31:591-7.

12.Register, A.A..Human bone induction by allogeneic dentin matrix. J Periodontol 1972; 43:459.

13.Dragoo M.R. Clinical and histologic evaluation of alloplasts and allografts in regenerative periodontal surgery in humans. Int J Periodont Rest Dent 1983; 3(2): 8 . 
14.Busschop J, \& De Boener, J. Clinical and histologic characteristics of lyophilized allogeneic duramater in periodontal bony defects in humans.J Clin. Periodontol 1982; 9: 141.

15.Carranza F.A. \& Newman M.G. Clinical periodontology, eighth edition, W.B. Saunders Company, 1996; 622-39.

16.Chodroff, R.E. \& Ammons, W.F. Periodontal repair and surgical debridement with and without cartilage allografts. J Clin. Periodontol 1984;11:295.

17.Alderman NE. Sterile plaster of Paris as an implant in the infrabony environment. A preliminary study.J Periodontol 1969; 40: 11.

18.Aichelmann - Reidy ME \& Yukna R.A. Bone replacement grafts. The bone substitutes. Dent. Clin. North
Am. Advances in Periodontics 1998;Part II 42(3): 491-501.

19.Mellonig, Bowers G.M. Comparison of bone graft materials. (Part I) J Periodontol 1981; 52: 291.

20.Genco, Goldman, Cohen. Contemporary periodontic. 1st Edition. The C.V.Mosby Company,1990; 585-604.

How to cite this article:

Tatuskar P, Prakash S. Bone Grafts in Periodontics

-A Review. CODS J Dent 2015;7: 64-70.

Source of support: Nil. Conflict of interest: None Declared. 